\title{
Perioperative use of intra-articular steroids during the COVID-19 pandemic
}

\author{
Eric Jou ${ }^{1,2} \odot \cdot$ Andrew Kailin Zhou ${ }^{1} \cdot$ Jamie Sin Ying $\mathrm{Ho}^{3} \cdot$ Azeem Thahir ${ }^{1,4}$
}

Received: 29 June 2021 / Accepted: 23 August 2021 / Published online: 1 September 2021

(c) The Author(s) 2021

\begin{abstract}
Purpose There are growing concerns with the widely used glucocorticoids during the Coronavirus disease-19 (COVID19) pandemic due to the associated immunosuppressive effects, which may increase the risk of COVID-19 infection and worsen COVID-19 patient outcome. Heavily affecting orthopaedics, the pandemic led to delay and cancellation of almost all surgical cases, and procedures including perioperative intra-articular corticosteroid injections (ICIs) saw similar decreases. However, the benefits of ICI treatments during the pandemic may outweigh these potential risks, and their continued use may be warranted.

Methods A literature search was conducted, and all relevant articles including original articles and reviews were identified and considered in full for inclusion, and analysed with expert opinion. Epidemiological statistics and medical guidelines were consulted from relevant authorities.

Results ICIs allow a targeted approach on the affected joint and are effective in reducing pain while improving functional outcome and patient quality-of-life. ICIs delay the requirement for surgery, accommodating for the increased healthcare burden during the pandemic, while reducing postoperative hospital stay, bringing significant financial benefits. However, ICIs can exert systemic effects and suppress the immune system. ICIs may increase the risk of COVID-19 infection and reduce the efficacy of COVID-19 vaccinations, leading to important public health implications.

Conclusion Perioperative ICI treatments may bring significant, multifaceted benefits during the pandemic. However, ICIs increase the risk of infection, and perioperative COVID-19 is associated with mortality. The use of ICIs during the COVID-19 pandemic should therefore be considered carefully on an individual patient basis, weighing the associated risks and benefits.
\end{abstract}

Keywords Injections $\cdot$ Intra-articular $\cdot$ Perioperative period $\cdot$ Steroids $\cdot$ SARS-CoV-2 $\cdot$ COVID-19 $\cdot$ Pandemics

\section{Introduction}

The use of intra-articular corticosteroid injections (ICIs) started in the early 1950s [1] and is now routinely used for management of joint pain and therapeutic treatment across a broad range of conditions [2-4]. ICI treatments allow

Eric Jou

ej290@cam.ac.uk

1 School of Clinical Medicine, University of Cambridge, Cambridge, UK

2 MRC Laboratory of Molecular Biology, Cambridge Biomedical Campus, Cambridge, UK

3 North Middlesex University NHS Trust, London, UK

4 Department of Trauma and Orthopaedic Surgery, Addenbrooke's Hospital, Cambridge University Hospitals NHS Foundation Trust, Cambridge, UK symptomatic relief and show therapeutic efficacy when administered to almost all major areas of the body, including the joints of the upper limb (shoulder, elbow, wrist and hand), lower limb (knee, ankle and foot), facet joints of the spine, the sacroiliac joint, the coccyx, and the hip, leading to its widespread use with broad implications [4]. In particular, perioperative ICIs are commonly used for pain relief, allowing better recovery of function postoperatively and improved patient quality-of-life $[5,6]$. Perioperative is defined as the medical care from the time of considering surgery through to the postoperative period where the patient strives to obtain a full recovery (preoperative, intraoperative and postoperative) [7]. Nevertheless, due to the potential danger of infections associated with steroid-mediated immunosuppression [8-12], the contemporary COVID-19 pandemic has instigated significant additional risks that need to be addressed when considering ICI use. 
The Coronavirus disease-19 (COVID-19) pandemic is caused by the severe acute respiratory syndrome coronavirus 2 (SARS-CoV-2), a novel coronavirus transmitted via respiratory droplets, and was first identified in late 2019 in Wuhan, China [13]. As estimated by the WHO, there are more than 110 million confirmed cases globally, with 2.5 million deaths worldwide as of February 2021 [14]. Disease severity in COVID-19 patients ranges from asymptomatic to life-threatening, with severe disease and mortality being more common in the elderly and in patients with underlying health conditions [15]. Furthermore, a significant proportion of COVID-19 patients may continue to experience long-term detrimental effects systemically, affecting the lungs, heart, brain, and the immune system [16], several weeks or months after the initial infection. The pandemic imposed an unprecedented burden on healthcare worldwide, forcing healthcare provision and practice to adapt globally [17-19]. In particular, the use of immunosuppressive agents, including the widely used corticosteroids, may heighten the risk of viral infections and increase COVID-19 severity [15, 20-23]. Perioperative use of ICIs must be carefully evaluated, and the impact on patient outcome needs to be established.

Surgical procedures have been particularly affected by the COVID-19 pandemic [24, 25]. Many countries made the difficult decision to postpone or cancel most elective surgeries, to better direct medical personnel and resources to combat the pandemic [26, 27]. In particular, orthopaedic procedures have been heavily impacted. Over a 12 -week period of peak healthcare disruption, a projected 28.4 million surgical operations would be cancelled globally, of which 6.3 million are non-emergency orthopaedic surgeries [24]. Orthopaedics is predicted to be the most heavily affected surgical specialty worldwide, with the highest cancellation rate (at $82 \%$ ) out of all surgical specialties. Hospital beds are prioritised for COVID-19 patients, and surgical trainees are redeployed to reinforce medical specialties in treating COVID-19 patients [28], including orthopaedic trainees across the UK [29, 30]. As of February 2021, 10 million patients are awaiting surgery in the UK, a dramatic increase from 4 million pre-pandemic [26]. Recognising the growing concerns of large-scale cancellation of non-emergency procedures [31, 32], elective surgeries had resumed since the latter half of 2020, albeit at much reduced capacities [26]. Healthcare services, and how perioperative care is delivered, had to adapt accordingly to provide the best care for patients. Perioperative ICIs may play an important role in improving symptom control and patient quality-of-life during the delay to elective surgeries, for example for elective joint replacements commonly performed for arthritis. Importantly, reassessing the safety and efficacy of these common perioperative management strategies employed prior to the pandemic, including ICIs, to adapt to the current pandemic is paramount.
The aim of this article was to review the benefits and risks associated with perioperative ICIs during the COVID-19 pandemic in light of recent evidence, with specific focus on the impact by the pandemic, along with medical, financial and public health implications. The potential interactions between ICIs and the immune response against SARSCoV-2 is also discussed and is of high relevance with important impact on the ongoing COVID-19 vaccination programme.

\section{Methods}

A literature search was first conducted on PubMed in January 2021, and again in February 2021, and all the relevant articles in the English language, including original articles and reviews, were identified. All non-peer-reviewed articles were excluded. Key search terms used were "steroid", "intra-articular injection", "COVID-19", "SARS-CoV2", "pandemic", "virus", "immune", and "vaccine". No articles were excluded based on publication date. A serial screening method was adopted due to the large number of candidate articles. Initially, article titles were screened for relevance to the factors discussed in this narrative review, followed by a second round of screening through the abstracts. Relevant articles were then considered in full, and the references cited in these articles were additionally screened, before inclusion. The World Health Organization (WHO) website was consulted for up-to-date global statistics regarding the COVID-19 pandemic and recommended guidelines on steroid use during the pandemic. The UK government website, and websites of relevant healthcare bodies including the National Health Service (NHS), the National Institute for Health and Care Excellence (NICE), were consulted for COVID-19 pandemic-related healthcare statistics, healthcare guidelines, and details on the UK COVID-19 vaccination programme. The PRISMA flow diagram for the literature search is presented in the supplementary material.

\section{ICl treatments during the COVID-19 pandemic}

ICI treatments involve the administration of glucocorticoids, a class of corticosteroids that binds to the glucocorticoid receptor. Glucocorticoids were first used therapeutically in humans in 1948 to treat rheumatoid arthritis (RA) [33], and ICIs are now commonly used in clinical practice [34]. Clinically administered glucocorticoids are similar in structure and function to endogenous cortisol, exerting potent anti-inflammatory effects in a dose-dependent manner [35]. Due to their widespread action on various cell types, clinical use of glucocorticoids has been limited by the broad range of potential adverse effects. In particular, the pioneering study by Fauci et al. in the 1970s identified that glucocorticoid administration leads to lymphopenia and 
immunosuppression [36], and steroid use is associated with increased risk of infection both in the community and in hospital settings [8-12]. Importantly, the increased risk of infection is not limited to systemic steroid use, with growing evidence demonstrating that local administration of steroids also heightens infection risk [37, 38]. Accordingly, in patients with rotator cuff tendinosis, a single corticosteroid injection up to a month prior to surgery increased the risk of postoperative infections twofold [37]. The use of steroids in the contemporary COVID-19 pandemic thus needs to be exercised with caution.

Regulating bodies including the National Health Service (NHS), the British Orthopaedic Association, and the Faculty of Pain Medicine, have raised concerns on the immunosuppressive effects of corticosteroids, issuing specific guidance accordingly to limit the use of steroid injections during the COVID-19 pandemic [39-41]. Clinicians are advised to maintain awareness of the risks associated with corticosteroid injections during the COVID-19 pandemic, and to assess the risk to benefit ratio for each patient on an individual basis. Specifically, ICIs should only be used in inflammatory conditions where there is active synovitis and at the lowest clinical effective dose. For musculoskeletal pain management, ICIs may only be used if other methods, including simple analgesia, splinting, active modification, and exercise prove ineffective, in addition to a high degree of pain and disability projected to have a significant impact on patient health and wellbeing [40]. Strictly, injected steroids should not be administered in the presence of an infection.

The guidance is welcomed, as studies suggest steroids are often misused clinically without definitive evidence on safety and efficacy [42, 43]. Often underappreciated, local administration of steroids can exert systemic effects [44], where a single dose of $40 \mathrm{mg}$ of methylprednisolone is sufficient for complete hypothalamic-pituitary-adrenal (HPA) suppression. This is well within the range of 4-80 mg methylprednisolone routinely used clinically through ICIs [45]. Others have similarly found that a single dose of ICI can suppress the HPA axis systemically [46, 47]. Strikingly, increased duration of systemic suppression is observed after ICI treatment compared to oral steroids, due to the slower rate of absorption through joint tissue [48]. A $46 \%$ mean decrease in both C-reactive protein (CRP) and erythrocyte sedimentation rate (ESR) was observed in RA patients after knee ICI treatment, with the effect lasting up to 6 months [49]. Independent studies had found a similar decrease in CRP or ESR levels in RA patients post ICI treatment [50, 51], suggesting systemic anti-inflammatory effects of ICIs. In line with this, ICIs decrease pro-inflammatory cytokines and inhibit monocyte function systemically $[52,53]$ and increase the risk of infection after knee, hip and shoulder arthroscopy [38]. Given that ICIs may lead to systemic immunosuppression and increase risk of infection, it should be used with caution clinically, more so amid the COVID-19 pandemic. Further studies are required to better understand the interactions between ICI-mediated immunosuppression and COVID-19 infections.

\section{ICls and the immune response against SARS-CoV-2}

The immune response against SARS-CoV-2 is complex (Fig. 1), and despite the rapid advances in recent months, much remains to be clarified. Accumulating evidence suggests that both the innate and the adaptive immune systems play crucial roles in the defence against SARS-CoV-2 [54], and better understanding of these processes is vital to improve patient care and outcome amid the pandemic. Specifically, it is crucial to elucidate the impact of corticosteroids on the immune response against SARS-CoV-2.

The immune response is effective in clearing SARSCoV-2 infections in most patients, with an overall estimated death rate of $0.66 \%$ [55]. However, mortality rises sharply to $40 \%$ in patients with severe COVID-19 [56], where abnormal immune hyperactivation inflicts indirect host damage through widespread lung inflammation and multiorgan damage [54]. The reason behind this divergence in patient immune response against SARS-CoV-2 is unclear, but likely relates to the immune dysregulation observed in severe COVID-19 patients. Increase in inflammatory monocytes correlates with severity of disease [57, 58], and recruitment is through chemokines CCL2 and CCL7, both of which elevated in fatal COVID-19 cases [59]. Monocytes directly contribute to the cytokine storm profile observed in severe COVID-19 patients, indicated by a systemic increase in interleukin-6 (IL-6), interleukin-8 (IL-8), interferon- $\gamma$ inducible protein 10 (IP-10), monocyte chemoattractant protein-1 (MCP1), macrophage inflammatory protein-1 alpha (MIP1 $\gamma$, and TNF $[54,60]$. In line with this, anti-inflammatory therapies through immunosuppressive glucocorticoids decrease mortality in severe COVID-19 patients and should be used [61, 62]. Any non-emergency surgery for patients that develop severe COVID-19 must be postponed and only proceed when medically safe to do so.

While steroid use is advocated in severe COVID-19 cases [63], its use in non-COVID-19 patients or in mild and moderate COVID-19 cases should be exercised with caution, due to the immunosuppressive effects as indicated by the WHO [64]. In mild and moderate COVID-19 patients, steroid use correlates with prolonged duration of fever, delayed virus clearance time, severe disease and longer hospitalisation [65-68]. Mechanistically, antigen-specific type 1 immunity driven by Thelper type 1 (Th1) and CD8 T cells are paramount in mounting a strong multimodal immune response against SARS-CoV-2 (Fig. 1), characterised by robust interferon- $\gamma(\mathrm{IFN} \gamma)$ production and a strong B cellmediated antibody response $[57,69]$. Conversely, patients 


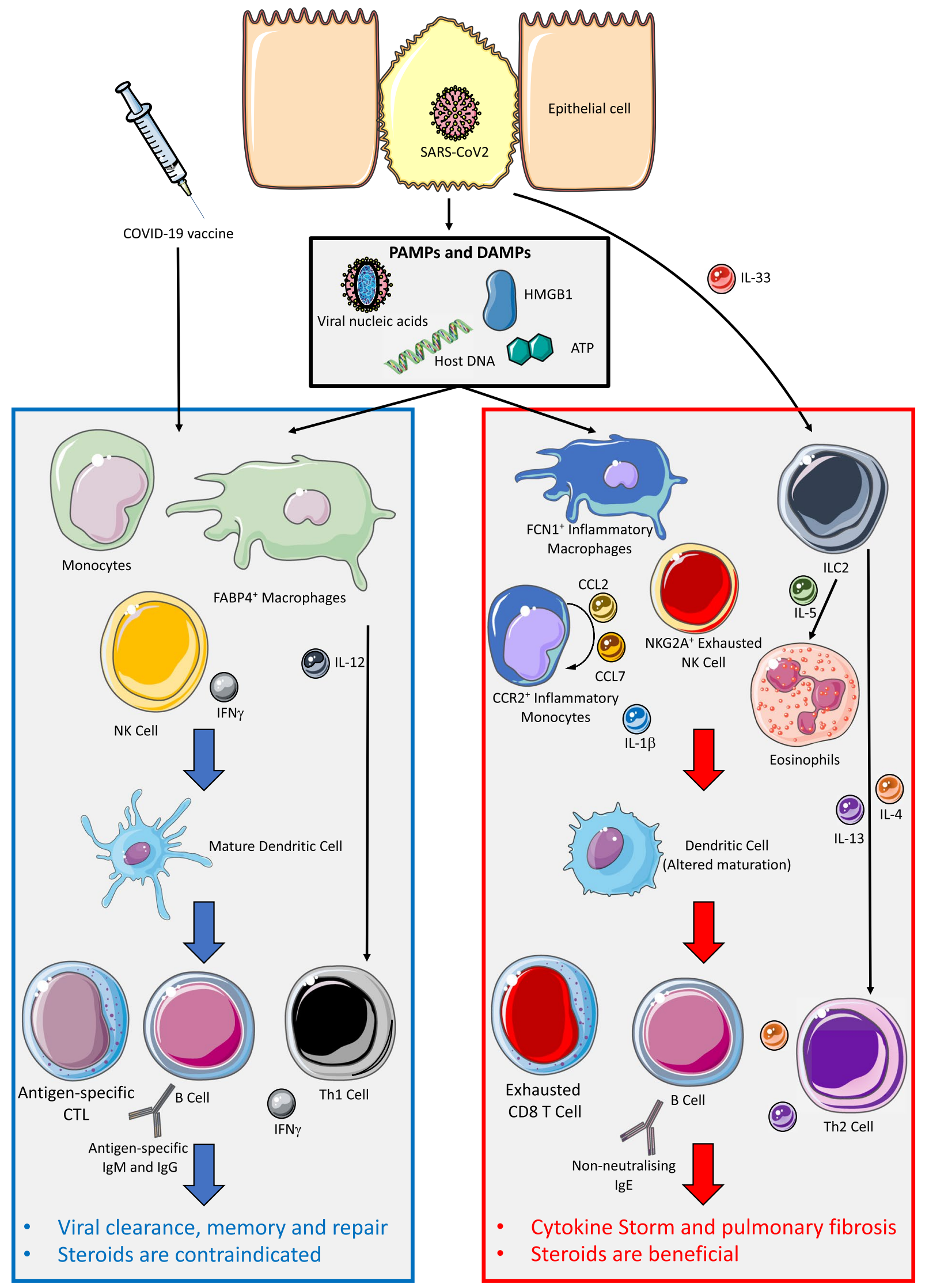


४Fig. 1 The immune response dichotomy against SARS-CoV2 infection in airway epithelial cells leads to cell death and the release of damage-associated molecular patterns (DAMPs), including adenosine triphosphate (ATP), DNA, and High Mobility Group Box 1 (HMGB1), the latter elevated in COVID-19 patients and correlates with inferior outcome. DAMPs and SARS-CoV2-derived pathogenassociated molecular patterns (PAMPs) including viral nucleic acids are detected by tissue resident innate immune cells such as macrophages, leading to cytokine production and recruitment of monocytes and NK cells which further amplifies the response. In mild and moderate COVID-19 patients (left blue panel), FABP4 ${ }^{+}$alveolar macrophages and anti-viral NK cells produce IL-12 and interferon$\gamma \mathrm{IFN} \gamma$, respectively, and together with mature dendritic cells, promote CD4 $\mathrm{T}$ cell $\mathrm{T}$ helper type 1 (Th1) polarisation and activation of antigen-specific CD8 cytotoxic T lymphocytes (CTLs), leading to elimination of viral-infected cells. B cells produce antigen-specific $\mathrm{IgM}$ and $\mathrm{IgG}$, which are effective in neutralising the virus, and together, this is characteristic of an effective anti-viral type 1 immune response, allowing viral clearance and development of immunological memory. A similar response is observed after COVID-19 vaccination in non-COVID-19 patients. In severe COVID-19 patients (right red panel), an inappropriately heightened immune response leads to a significant increase in CCR2 ${ }^{+}$inflammatory monocytes and $\mathrm{FCN}{ }^{+}$inflammatory macrophages, replacing the $\mathrm{FABP}^{+}$alveolar macrophages seen in non-severe patients. A positive-feedback loop through increased production of chemokines CCL2 and CCL7 further recruits inflammatory monocytes and promote the production of pro-inflammatory cytokine IL- $1 \gamma$. In these patients, NK cells show an exhausted phenotype, characterised by NKG2A expression, and have impaired anti-viral function. Additionally, widespread epithelial damage releases the alarmin IL-33, leading to the characteristic increase in group 2 innate lymphoid cells (ILC2s) seen in severe COVID-19 patients. ILC2s are potent producers of IL-4, IL-5 and IL-13. IL-5 recruits and activates eosinophils, and increased eosinophils contribute to the inflammatory pathology and correlates with adverse outcomes in COVID-19 patients. Altered dendritic cell maturation, together with the IL- 4 and IL-13 produced by ILC2s, lead to altered CD4 $\mathrm{T}$ cell activation and subsequent polarisation towards a $\mathrm{T}$ helper type 2 (Th2) phenotype with impaired anti-viral functions. Th2 cells further produces IL-4 and IL-13, which promotes B cell antibody class switch to $\operatorname{IgE}$, and the non-neutralising antibodies may facilitate SARS-CoV2 infection via antibody-dependent enhancement. CD8 $\mathrm{T}$ cells show an exhausted phenotype with defective anti-viral functions. Together, the heightened inflammatory environment and inadequate viral clearance can result in irreversible pulmonary fibrosis and perpetuate a cytokine storm, leading to multi-organ damage. In severe COVID-19 patients, steroids reduce mortality by dampening the inappropriately hyperactivated immune response and cytokine storm, while its use is contraindicated in mild and moderate COVID19 patients where suppression of the protective anti-viral immune response may increase disease severity. In non-COVID patients, steroids may increase the risk of infection and decrease the efficacy of COVID-19 vaccinations [54, 57-59, 64, 69, 70, 99, 102-105]. This figure was created using templates by Servier Medical Art, licensed under a Creative Commons Attribution 3.0 Unported License (https:// smart.servier.com/)

that develop severe COVID-19 have decreased T cells, and T cells show an exhausted phenotype with impaired functions [70]. Reduced lymphocyte count is a negative prognostic indicator in COVID-19 patients [71]. Given that a single dose of ICI reduces systemic blood lymphocyte count, leading to lymphopenia four hours after injection [52], ICIs may impair the host protective response against SARS-CoV-2. The systemic and immunosuppressive effects of steroid injections may last several weeks [72] and could consequentially expose patients to COVID-19 infection and developing severe disease. Accordingly, ICIs increases the risk of influenza even among vaccinated individuals [23]. In this study, the average dose patient received through a single ICI was $65.9 \mathrm{mg}$, with greater than half of the patients receiving $>80 \mathrm{mg}$ of methylprednisolone or triamcinolone acetonide or equivalent, which is well within the range to cause systemic effects [44]. Critically, the risk of patients acquiring COVID-19 infection perioperatively should be minimised, as perioperative SARS-CoV-2 infection increases mortality in both elective and emergency surgical patients [73]. The National Institute for Health and Care Excellence (NICE) published guidelines for elective patients to follow comprehensive social-distancing measures for 14 days, and to test for SARS-CoV-2 within 3 days prior to hospital admission [74]. The threshold for surgery should be heightened during the COVID-19 pandemic, and use of perioperative ICI must be carefully evaluated. The increased risk associated with COVID-19 infection must be balanced with the consequences of delaying elective surgeries, and the use of ICIs should be discussed in conjunction with patients on an individual basis.

\section{Impact on patient quality-of-life during the COVID-19 pandemic}

In light of the adverse effects of systemic glucocorticoid treatments, ICI is popular as it allows a localised approach with targeted effect on the affected joint [75]. Perioperative ICIs are common for a wide range of orthopaedic surgeries, both pre- and postoperatively.

\section{Preoperative use of ICI during COVID-19: delaying elective surgery}

In osteoarthritis $(\mathrm{OA})$ patients requiring surgery, preoperative ICIs are associated with a delay to TKA from first presentation to clinic by 8 months, and a similar 5-month delay for patients that received total hip arthroplasty [76]. This is attributed to preoperative ICIs providing comfort, symptom alleviation and reducing functional disability. Overall, the benefits of the delay in time to surgery from initial clinical presentation are twofold. Firstly, it allows patients time to prepare for surgery mentally and to make necessary arrangements for social support. Secondly, and specifically related to the COVID-19 pandemic where elective surgeries are postponed, preoperative ICIs are effective in improving patient quality-of-life during the delay to surgery. Reassuringly, studies of patients given ICI during the COVID-19 pandemic in the UK did not show any increase in COVID-19 
infection or adverse effects [77, 78]; therefore, ICI may be a safe and viable option. Of note, while ICI clinics may also work at reduced capacities during the pandemic, reinstating ICI clinics could be a practical and more feasible approach to combat the significant delays in elective surgeries.

\section{Postoperative use of ICI during COVID-19: post-operative pain management}

Postoperative ICIs reduce pain and swelling and improve range of motion after TKA [6]. Improving patient quality-oflife is paramount, as $50 \%$ of patients suffering from chronic pain have associated psychological distress [79]. OA is one of the top ten most debilitating diseases worldwide [80], causing significant pain with $60 \%$ of patients awaiting knee replacement surgery showing signs of clinical depression [26]. Clinical depression may further rise to $90 \%$ in patients suffering from orthopaedic-related axial pain. Patients diagnosed with mental disorders have increased risk of acquiring COVID-19 [81] and are more likely to develop severe disease [82]. Furthermore, severe postoperative pain is associated with suppressed immune function, and may predispose to infection [83]. Use of ICIs may improve patient health both physically and mentally and thus achieve better quality-of-life amid the pandemic and should be considered after discussing and assessing each patient and their specific circumstances on an individual basis.

There is an inherent fear for a potential rise in self harm or suicide rate during the COVID-19 pandemic. While current existing reports had found no increase in suicide rates in relation to the pandemic [84], it is essential to remain vigilant, as a potential increasing trend in certain vulnerable groups may be masked by an overall unchanging suicide rate. Strikingly, patients with arthritis have significantly increased risk for suicide with $46 \%$ higher odds than those without arthritis [85]. Among the arthritis patients, those currently experiencing chronic pain had increased risk of suicide compared to those without pain (odds ratio of 1.5). The delay in elective surgeries due to the pandemic is unprecedented and can lead to prolonged suffering of pain in arthritis patients awaiting joint replacement surgeries. The American Academy of Orthopaedic Surgeons had suggested that preoperative ICIs should be considered for pain management in surgical arthritis patients during the delay to surgery [86]. The use of ICIs can improve pain management and may be effective in reducing suicide rates in arthritis patients. Extended suffering during the delay to surgery for elective patients should not be forgotten, and increasing the capacity of ICI clinics during the pandemic should be considered as a feasible temporary solution. Better symptomatic control and pain management is more important than ever amid the pandemic, and ICIs are an effective treatment modality that can be employed to achieve this.

\section{Financial implications of perioperative ICls amid the COVID-19 pandemic}

The COVID-19 pandemic had imposed significant financial burden on healthcare systems worldwide, with an estimated $£ 40$ billion extra healthcare service cost per year on the NHS [87]. The cost-effectiveness of treatment modalities needs to be considered. A recent UK-based study concluded that addition of ICI and lidocaine to standard hip OA treatment significantly improved function and incurred less cost (£161.59 per patient) over a 6-month period [88], attributed to the higher quality-adjusted life-years. In a randomised control trial (RCT), sonographic guidance for knee ICIs reduced procedural pain, doubled the responder rate, increased the therapeutic duration by $36 \%$ while reducing cost by $58 \%$ in responders per year [89]. Use of sonographic guidance over traditional anatomy landmark methods during ICIs directly improves clinical outcome and cost-effectiveness and should be used whenever appropriate [90]. Another UK study found steroid injections to be cost-effective compared to physiotherapy, with an average saving of $£ 43.32$ per patient while outcomes were similar [91]. Alternatively, intra-articular injection of ketorolac produced similar degree of pain relief to ICI while saving cost in a small study and may be considered [92]. Strikingly, perioperative corticosteroid injection prior to TKA shortens mean hospital stay by $25 \%$ in a RCT [93]. This is desirable as reducing hospitalisation time decreases the risk of hospital-acquired COVID-19, accommodates for the increased medical demand amid the pandemic [94], and has important financial implications on cost-savings from reduced hospital stay $[95,96]$.

\section{Public health implications of $\mathrm{ICl}$ treatments on the COVID-19 vaccination programme}

Global roll-out of COVID-19 vaccines plays a critical role in combating the pandemic. The comprehensive, tiered UK vaccination programme prioritises the most clinically vulnerable, to protect those that are at highest risk of severe COVID-19 outcomes [97]. Factors associated with worse COVID-19 prognosis, including reduced physical activity, increased age and body mass index, are associated with orthopaedic conditions such as OA where ICIs are routinely used [98]. Of concern, ICIs may reduce the efficacy of COVID-19 vaccinations at clinically used doses [99], due to the potential immunosuppressive effects on the protective type 1 immune response (Fig. 1), observed both in natural COVID-19 infections [57] and after COVID19 vaccination [69]. While more studies are required to assess the specific effects of ICIs on COVID-19 vaccinations, previous studies identified ICIs to reduce the efficacy of influenza vaccination [23]. Suboptimal response to COVID-19 vaccination will have significant negative 
Fig. 2 Implications of intra-articular corticosteroid injections amid the Coronavirus disease-19 (COVID-19) pandemic. The positive and negative implications of ICI treatment during the COVID-19 pandemic, along with recent guidelines by relevant authorities, are summarised in the infographic

\section{Pros and Cons of \\ Perioperative Intra-articular \\ Corticosteroid Injections (ICI) Treatment Amid the COVID-19 Pandemic}

\section{Potential positive implications of ICI treatment during the COVID-19 pandemic}

Improve patient quality of life

Better pain management

- Improve functional outcome postoperatively

- May reduce suicide risk

May reduce mental health disorders

Financial benefits

Reduce hospitalisation time

Cost-effective

Delay the need of surgery from initial clinical presentation

Allows patients time to prepare for surgery mentally, and to organise necessary social support

- Help accommodate the increase in medical burden during the pandemic

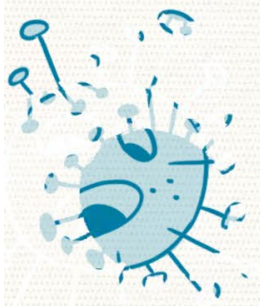

\section{Potential negative implications of $\mathrm{ICl}$ treatment during} the COVID-19 pandemic

Increase risk of infection

In non-severe COVID-19 patients, steroids may increase

hospitalisation and viral shedding time

May reduce COVID-19 vaccine efficacy if administered within two weeks

May facilitate virus evolution and appearance of vaccine escape strains

Immunocompromised patients may be less responsive to current COVID-19 therapies eg. Remdesivir

\section{Guidelines on ICI treatments during COVID-19 pandemic}

NHS guidelines

- Clinicians should maintain awareness of the risks associated with steroid injections amid the pandemic, and assess the risk: benefit ratio for each patient on an individual basis.

- ICls should only be used in inflammatory conditions where there is active synovitis and at the lowest clinical effective dose.

- For musculoskeletal pain management, ICls may only be used if other methods, including simple analgesia, splinting, active modification, and exercise prove ineffective, in addition to a high degree of pain and disability projected to have a significant impact on patient health and wellbeing.

- Injected steroids should not be administered in the presence of an infection.

WHO

- Steroids should not be administered in non-severe COVID-19 patients as it may cause harm.

AAOS

- ICls should be considered for pain management in surgical arthritis patients during the delay to surgery.

ARMA (in conjunction with BOA, BSR and RAIRDA)

- COVID-19 vaccinations should take priority, and any non-essential steroid injections should be deferred by 2 weeks to allow better response to the vaccine. 
public health implications and should be avoided at all costs. Current guidance in the UK suggests that for elective patients, COVID-19 vaccinations should take priority over any form of steroid injections including ICIs, which is advised to be postponed by 2 weeks to allow optimal response to the COVID-19 vaccine [99]. Finally, it has been suggested that evolution of SARS-CoV2 may be accelerated in immunosuppressed individuals, due to the suboptimal immune response allowing viral persistence [100]. Furthermore, existing COVID-19 therapies, such as remdesivir, may be less effective in eradicating SARS$\mathrm{CoV} 2$ in immunosuppressed individuals leading to persistent infections [101], which may potentially facilitate the appearance of vaccine escape strains.

\section{Conclusion}

In accordance to recent guidance from the NHS, the threshold for perioperative ICIs should be heightened amid the COVID-19 pandemic. Although administered locally, ICIs exert systemic anti-inflammatory and immunosuppressive effects at clinically relevant doses and increase the risk of perioperative infections. Importantly, perioperative SARS-CoV-2 infection should be avoided due to the associated increase in mortality. ICIs may reduce the efficacy of COVID-19 vaccinations, which can have significant public health impacts. Equally, while capacities for elective ICI procedures during the pandemic may be reduced, reinstating perioperative ICIs to improve pain management and patient quality-of-life is suggested by the American Academy of Orthopaedic Surgeons. ICI treatments may delay the need for surgery, while enhancing functional outcome postoperatively, and may be cost-effective with important financial implications. Arthritis patients have increased risk of suicide, and suicide rate is further increased in those suffering from chronic pain. The delay in elective surgeries instigated by the pandemic may prolong patient suffering in those awaiting joint replacement surgeries. ICIs may allow better symptomatic control and pain management during the delay. Of note, poorly managed postoperative pain is associated with immunosuppression and worse functional outcome. Clinical decisions on the use of ICIs should thus be made on an individual basis, and discussed with patients to achieve the best overall outcome (Fig. 2). It is paramount that flexibilities exist in the guidelines for ICI treatments to best accommodate for individual patient circumstances. Future research will provide further evidence to better evaluate the safety of ICI treatments during the COVID-19 pandemic.
Supplementary Information The online version contains supplementary material available at https://doi.org/10.1007/s00590-021-03105-x.

Acknowledgements This study received no external funding, and the authors declared no competing interests.

\section{Declarations}

Conflicts of interest The authors declare no conflicting interest.

Data Availability Data available upon request.

Open Access This article is licensed under a Creative Commons Attribution 4.0 International License, which permits use, sharing, adaptation, distribution and reproduction in any medium or format, as long as you give appropriate credit to the original author(s) and the source, provide a link to the Creative Commons licence, and indicate if changes were made. The images or other third party material in this article are included in the article's Creative Commons licence, unless indicated otherwise in a credit line to the material. If material is not included in the article's Creative Commons licence and your intended use is not permitted by statutory regulation or exceeds the permitted use, you will need to obtain permission directly from the copyright holder. To view a copy of this licence, visit http://creativecommons.org/licenses/by/4.0/.

\section{References}

1. Hollander JL, Brown EM Jr, Jessar RA et al (1951) Hydrocortisone and cortisone injected into arthritic joints; comparative effects of and use of hydrocortisone as a local antiarthritic agent. J Am Med Assoc 147(17):1629-1635

2. Arroll B, Goodyear-Smith F (2004) Corticosteroid injections for osteoarthritis of the knee: meta-analysis. BMJ 328(7444):869-869

3. Kokubun BA, Manista GC, Courtney PM et al (2017) Intraarticular knee injections before total knee arthroplasty: outcomes and complication rates. J Arthroplasty 32(6):1798-1802

4. Stephens MB, Beutler AI, O'Connor FG (2008) Musculoskeletal injections: a review of the evidence. Am Fam Physician 78(8):971-976

5. Donohue NK, Prisco AR, Grindel SI (2017) Pre-operative corticosteroid injections improve functional outcomes in patients undergoing arthroscopic repair of high-grade partial-thickness rotator cuff tears. Muscles Ligaments Tendons J 7(1):34-39

6. Klement MR, Luzzi AJ, Siddiqi A et al (2019) Intra-articular corticosteroid injection following total knee arthroplasty: is it effective? J Arthroplasty 34(2):303-308

7. Schonborn JL, Anderson H (2019) Perioperative medicine: a changing model of care. BJA Edu 19(1):27-33

8. Chaudhary NS, Donnelly JP, Moore JX et al (2017) Association of baseline steroid use with long-term rates of infection and sepsis in the REGARDS cohort. Crit Care 21(1):185

9. Hoes JN, Jacobs JW, Verstappen SM et al (2009) Adverse events of low- to medium-dose oral glucocorticoids in inflammatory diseases: a meta-analysis. Ann Rheum Dis 68(12):1833-1838

10. Dixon WG, Kezouh A, Bernatsky S et al (2011) The influence of systemic glucocorticoid therapy upon the risk of non-serious infection in older patients with rheumatoid arthritis: a nested case-control study. Ann Rheum Dis 70(6):956-960 
11. Fardet L, Petersen I, Nazareth I (2016) Common infections in patients prescribed systemic glucocorticoids in primary care: a population-based cohort study. PLoS Med 13(5):e1002024

12. Merkler AE, Saini V, Kamel H et al (2014) Preoperative steroid use and the risk of infectious complications after neurosurgery. Neurohospitalist 4(2):80-85

13. World Health Organization. Listings of WHO's response to COVID-19. 2020 [cited 2021 Feb 21]. Available from: https:// www.who.int/news/item/29-06-2020-covidtimeline

14. World Health Organization. WHO Coronavirus Disease (COVID-19) Dashboard. 2021

15. Zhou F, Yu T, Du R et al (2020) Clinical course and risk factors for mortality of adult inpatients with COVID-19 in Wuhan, China: a retrospective cohort study. The Lancet 395(10229): 1054-1062

16. Marshall M (2020) The lasting misery of coronavirus longhaulers. Nature 585(7825):339-341

17. Papoutsi E, Giannakoulis VG, Ntella V et al (2020) Global burden of COVID-19 pandemic on healthcare workers. ERJ Open Res 6(2):00195-02020

18. Miller IF, Becker AD, Grenfell BT et al (2020) Disease and healthcare burden of COVID-19 in the United States. Nat Med. 26(8):1212-1217

19. Aburto JM, Kashyap R, Schöley J, Angus C, Ermisch J, Mills MC et al (2021) Estimating the burden of the COVID-19 pandemic on mortality, life expectancy and lifespan inequality in England and Wales: a population-level analysis. J Epidemiol Commun Health 75(8):735-40. https://doi.org/10.1136/jech-2020-215505

20. Mishra GP, Mulani J (2021) Corticosteroids for COVID-19: the search for an optimum duration of therapy. Lancet Respir Med. 9(1):e8

21. Wang K, Zhang Z, Yu M et al (2020) 15-day mortality and associated risk factors for hospitalized patients with COVID-19 in Wuhan, China: an ambispective observational cohort study. Intensive Care Med 46(7):1472-1474

22. Wang Z, Yang B, Li Q et al (2020) clinical features of 69 cases with coronavirus disease 2019 in Wuhan. China Clin Infect Dis 71(15):769-777

23. Sytsma TT, Greenlund LK, Greenlund LS (2018) Joint corticosteroid injection associated with increased influenza risk. Mayo Clin Proc Innov Qual Outcomes 2(2):194-198

24. COVID Surg Collaborative (2020) Elective surgery cancellations due to the COVID-19 pandemic: global predictive modelling to inform surgical recovery plans. Br J Surg 107(11):1440-1449. https://doi.org/10.1002/bjs.11746

25. Al-Kulabi A, Mansour MA, Thahir A (2021) The orthopaedic experience of COVID-19: a literature review. J Perioper Pract 8:1750458920971506

26. The Lancet R (2021) Too long to wait: the impact of COVID-19 on elective surgery. The Lancet Rheumatol 3(2):e83-e. https:// doi.org/10.1016/S2665-9913(21)00001-1

27. Collaborative CO (2020) Global guidance for surgical care during the COVID-19 pandemic. Br J Surg 107(9):1097-1103

28. Payne A, Rahman R, Bullingham R et al (2020) Redeployment of surgical trainees to intensive care during the COVID-19 pandemic: evaluation of the impact on training and wellbeing. J Surg Educ S1931-7204(20):30359-30367

29. Gonzi G, Gwyn R, Rooney K et al (2020) The role of orthopaedic trainees during the COVID-19 pandemic and impact on postgraduate orthopaedic education. Bone Joint Open 1(11):676-682

30. Faria G, Tadros BJ, Holmes N et al (2020) Redeployment of the trainee orthopaedic surgeon during COVID-19: a fish out of water. Acta Orthopaedica 91(6):650-653

31. Stahel PF (2020) How to risk-stratify elective surgery during the COVID-19 pandemic? Patient Saf Surg 14:8
32. Pirracchio R, Mavrothalassitis O, Mathis M et al (2021) Response of US hospitals to elective surgical cases in the COVID-19 pandemic. British J Anaesthesia. 126(1):e46-e48

33. Benedek TG (2011) History of the development of corticosteroid therapy. Clin Exp Rheumatol. 29(5 Suppl 68):S5-S12

34. Martin SD, Conaway WK, Lei P (2018) Use of intra-articular corticosteroids in orthopaedics. J Bone Joint Surg Am 100(10):885-891

35. Buttgereit F, Da Silva JAP, Boers M et al (2002) Standardised nomenclature for glucocorticoid dosages and glucocorticoid treatment regimens: current questions and tentative answers in rheumatology. Ann Rheum Dis 61(8):718-722

36. Fauci AS, Dale DC, Balow JE (1976) Glucocorticosteroid therapy: mechanisms of action and clinical considerations. Ann Intern Med 84(3):304-315

37. Puzzitiello RN, Patel BH, Nwachukwu BU et al (2020) Adverse impact of corticosteroid injection on rotator cuff tendon health and repair: a systematic review. Arthroscopy 36(5):1468-1475

38. Haskel JD, Kaplan DJ, Kirby DJ, et al. Revisiting Intraarticular Corticosteroid Injections and Sports Medicine: Outcomes and Perioperative Considerations. SN Comprehensive Clinical Medicine. 2021 2021/01/21

39. British Orthopaedic Association. Corticosteroid use for musculoskeletal and rheumatic conditions during COVID-19 pandemic. 2020 [cited 2021 Feb 15]. Available from: https://www. boa.ac.uk/uploads/assets/30a67bae-1e3a-4b76-bf97b7f866 00230b/Corticosteroid-use-for-musculoskeletal-and-rheum atic-conditions-during-COVID-19-Pandemic-V1.pdf

40. National Health Service England. Clinical guide during the COVID-19 pandemic for the management of patients with musculoskeletal and rheumatic conditions. 2020 [cited 2021 Feb 11]. Available from: https://www.rheumatology.org.uk/ Portals/0/Documents/COVID-19/MSK_rheumatology_corti costeroid_guidance.pdf

41. College of Podiatry. Steroid injections and COVID-19 Updated Guidance November 2020. 2020 [cited 2021 Feb 10]. Available from: https://news.rcpsg.ac.uk/news/steroid-injec tions-and-covid-19-updated-guidance-november-2020/

42. Kompel AJ, Roemer FW, Murakami AM et al (2019) Intraarticular corticosteroid injections in the hip and knee: perhaps not as safe as we thought? Radiology 293(3):656-663

43. Marsland D, Mumith A, Barlow IW (2014) Systematic review: the safety of intra-articular corticosteroid injection prior to total knee arthroplasty. Knee 21(1):6-11

44. Stout A, Friedly J, Standaert CJ (2019) Systemic absorption and side effects of locally injected glucocorticoids. Pm r 11(4):409-419

45. Excellence NIfHaC. Methylprednisolone 2021 [cited 2021 June 20]. Available from: https://bnf.nice.org.uk/drug/methylpred nisolone.html

46. Koehler BE, Urowitz MB, Killinger DW (1974) The systemic effects of intra-articular corticosteroid. J Rheumatol 1(1):117-125

47. Weitoft T, Rönnblom L (2006) Glucocorticoid resorption and influence on the hypothalamic-pituitary-adrenal axis after intra-articular treatment of the knee in resting and mobile patients. Ann Rheum Dis 65(7):955-957

48. Reeback JS, Chakraborty J, English J et al (1980) Plasma steroid levels after intra-articular injection of prednisolone acetate in patients with rheumatoid arthritis. Ann Rheum Dis 39(1):22-24

49. Taylor HG, Fowler PD, David MJ et al (1991) Intra-articular steroids: confounder of clinical trials. Clin Rheumatol 10(1):38-42

50. Emkey RD, Lindsay R, Lyssy J et al (1996) The systemic effect of intraarticular administration of corticosteroid on markers of 
bone formation and bone resorption in patients with rheumatoid arthritis. Arthritis Rheum 39(2):277-282

51. Pitsillides AA, Will RK, Bayliss MT et al (1994) Circulating and synovial fluid hyaluronan levels effects of intraarticular corticosteroid on the concentration and the rate of turnover. Arthritis Rheum. 37(7):1030-8

52. Steer JH, Ma DT, Dusci L et al (1998) Altered leucocyte trafficking and suppressed tumour necrosis factor alpha release from peripheral blood monocytes after intra-articular glucocorticoid treatment. Ann Rheum Dis 57(12):732-737

53. Alex P, Szodoray P, Arthur E et al (2007) Influence of intraarticular corticosteroid administration on serum cytokines in rheumatoid arthritis. Clin Rheumatol 26(5):845-848

54. Tay MZ, Poh CM, Rénia L et al (2020) The trinity of COVID-19: immunity, inflammation and intervention. Nature Rev Immunol. 20(6):363-374

55. Verity R, Okell LC, Dorigatti I et al (2020) Estimates of the severity of coronavirus disease 2019: a model-based analysis. Lancet Infect Dis 20(6):669-677

56. Armstrong RA, Kane AD, Cook TM (2020) Outcomes from intensive care in patients with COVID-19: a systematic review and meta-analysis of observational studies. Anaesthesia 75(10): 1340-1349

57. Lucas C, Wong P, Klein J et al (2020) Longitudinal analyses reveal immunological misfiring in severe COVID-19. Nature 584(7821):463-469

58. Liao M, Liu Y, Yuan J et al (2020) Single-cell landscape of bronchoalveolar immune cells in patients with COVID-19. Nat Med 26(6):842-844

59. Xu Z-S, Shu T, Kang L et al (2020) Temporal profiling of plasma cytokines, chemokines and growth factors from mild, severe and fatal COVID-19 patients. Signal Transduct Target Ther 5(1):100

60. Merad M, Martin JC (2020) Pathological inflammation in patients with COVID-19: a key role for monocytes and macrophages. Nat Rev Immunol 20(6):355-362

61. Wu C, Chen X, Cai Y et al (2020) Risk factors associated with acute respiratory distress syndrome and death in patients with coronavirus disease 2019 pneumonia in Wuhan. China JAMA Intern Med 180(7):934-943

62. Horby P, Lim WS, Emberson JR, et al. Dexamethasone in Hospitalized Patients with Covid-19 - Preliminary Report. N Engl J Med. 2020 Jul 17

63. Group TRC. Dexamethasone in Hospitalized Patients with Covid19. New England Journal of Medicine. 2020 384(8):693-704.

64. World Health Organization. WHO updates clinical care guidance with corticosteroid recommendations. 2020 [cited 2021 Feb 13]. Available from: https://www.who.int/news-room/feature-stories/ detail/who-updates-clinical-care-guidance-with-corticosteroidrecommendations

65. Budhathoki P, Shrestha DB, Rawal E, et al Corticosteroids in COVID-19: Is it Rational? A systematic review and meta-analysis. SN comprehensive clinical medicine. 2020:1-21

66. Shuto H, Komiya K, Yamasue M et al (2020) A systematic review of corticosteroid treatment for noncritically ill patients with COVID-19. Sci Rep. 10(1):20935

67. Li Q, Li W, Jin Y et al (2020) Efficacy evaluation of early, lowdose, short-term corticosteroids in adults hospitalized with non-severe COVID-19 Pneumonia: a retrospective cohort study. Infect Dis Ther 9(4):823-836

68. Arora K, Panda PK (2021) Steroid harms if given early in COVID-19 viraemia. BMJ Case Rep 14(2):e241105

69. Sahin U, Muik A, Derhovanessian E et al (2020) COVID-19 vaccine BNT162b1 elicits human antibody and T $\mathrm{H} 1 \mathrm{~T}$ cell responses. Nature. 586(7830):594-599
70. Zheng M, Gao Y, Wang G et al (2020) Functional exhaustion of antiviral lymphocytes in COVID-19 patients. Cell Mol Immunol 17(5):533-535

71. Wang D, Hu B, Hu C et al (2020) Clinical characteristics of 138 hospitalized patients with 2019 novel coronavirus-infected Pneumonia in Wuhan. China JAMA 323(11):1061-1069

72. Hackett BA (2020) Providing steroid/corticosteroid injections safely in the COVID-19 environment. J Radiol Nurs 39(3): 174-175

73. COVIDSurgCollaborative. Mortality and pulmonary complications in patients undergoing surgery with perioperative SARSCoV-2 infection: an international cohort study. The Lancet. 2020;396(10243):27-38

74. National Institute for Health and Care Excellence. NICE publishes new COVID-19 rapid guideline on arranging planned care in hospitals and diagnostic services. 2020 [cited 2021 Feb 14]. Available from: https://www.nice.org.uk/news/article/nice-publi shes-new-covid-19-rapid-guideline-on-arranging-planned-carein-hospitals-and-diagnostic-services

75. Wong SM, Hui AC, Tang A et al (2001) Local vs systemic corticosteroids in the treatment of carpal tunnel syndrome. Neurology 56(11):1565-1567

76. Tang A, Almetwali O, Zak SG et al (2021) Do preoperative intraarticular corticosteroid and hyaluronic acid injections affect time to total joint arthroplasty? J Clinic Orthop Trauma 16:49-57

77. Newton AC, Jones G, Jones JWM et al (2021) Intra-articular corticosteroid injections during the COVID-19 lockdown period: a service evaluation. Musculoskeletal Care 19(2):236-243

78. Bugeja M, Mariani J, Dowling J et al (2021) Musculoskeletal steroid injections during the COVID-19 pandemic. J Orthop 26:103-106

79. Manchikanti L, Fellows B, Singh V (2002) Understanding psychological aspects of chronic pain in interventional pain management. Pain Physician 5(1):57-82

80. Murray CJ, Vos T, Lozano R et al (2012) Disability-adjusted life years (DALYs) for 291 diseases and injuries in 21 regions, 1990-2010: a systematic analysis for the global burden of disease study 2010. Lancet 380(9859):2197-2223

81. Yang H, Chen W, Hu Y et al (2020) Pre-pandemic psychiatric disorders and risk of COVID-19: a UK Biobank cohort analysis. Lancet Healthy Longev 1(2):e69-e79

82. Lee SW, Yang JM, Moon SY et al (2020) Association between mental illness and COVID-19 susceptibility and clinical outcomes in South Korea: a nationwide cohort study. Lancet Psychiatr 7(12):1025-1031

83. Gan TJ (2017) Poorly controlled postoperative pain: prevalence, consequences, and prevention. J Pain Res 10:2287-2298

84. John A, Pirkis J, Gunnell D et al (2020) Trends in suicide during the covid-19 pandemic. BMJ 371:m4352

85. Fuller-Thomson E, Ramzan N, Baird SL (2016) Arthritis and suicide attempts: findings from a large nationally representative Canadian survey. Rheumatol Int 36(9):1237-1248

86. American Academy of Orthopaedic Surgeons. What to Do If Your Orthopaedic Surgery Is Postponed. 2020 [cited 2021 Feb 9]. Available from: https://orthoinfo.aaos.org/en/treatment/whatto-do-if-your-orthopaedic-surgery-is-postponed/

87. The Health Foundation. Spending Review 2020 - Priorities for the NHS, social care and the nation's health. 2020 [cited 2021 Feb 13]. Available from: https://www.health.org.uk/publicatio ns/long-reads/spending-review-2020

88. Paskins Z, Bromley K, Lewis M, Hughes G, Hughes E, Cherrington A et al (2020) Clinical and cost-effectiveness of ultrasound-guided intra-articular corticosteroid and local anaesthetic injection for hip osteoarthritis: a randomised controlled 
trial (HIT). Osteoarthritis Cartilage 28:S479. https://doi.org/10. 1016/j.joca.2020.02.751

89. Sibbitt WL Jr, Band PA, Kettwich LG et al (2011) A randomized controlled trial evaluating the cost-effectiveness of sonographic guidance for intra-articular injection of the osteoarthritic knee. J Clin Rheumatol 17(8):409-415

90. Berkoff DJ, Miller LE, Block JE (2012) Clinical utility of ultrasound guidance for intra-articular knee injections: a review. Clin Interv Ag 7:89-95

91. James M, Stokes EA, Thomas E et al (2005) A cost consequences analysis of local corticosteroid injection and physiotherapy for the treatment of new episodes of unilateral shoulder pain in primary care. Rheumatology 44(11):1447-1451

92. Bellamy JL, Goff BJ, Sayeed SA (2016) Economic impact of Ketorolac vs Corticosteroid intra-articular knee injections for osteoarthritis: a randomized, double-blind. Prospect Stud J Arthroplast 31(9 Suppl):293-297

93. Christensen CP, Jacobs CA, Jennings HR (2009) Effect of periarticular corticosteroid injections during total knee arthroplasty A double-blind randomized trial. J Bone Joint Surg Am 91(11):2550-2555

94. Wang L, Lu X, Zhang J et al (2020) Strategies for perioperative management of general surgery in the post-COVID-19 era: experiences and recommendations from frontline surgeons in Wuhan. Br J Surg 107(10):e437

95. Fine MJ, Pratt HM, Obrosky DS et al (2000) Relation between length of hospital stay and costs of care for patients with community-acquired pneumonia. Am J Med 109(5):378-385

96. National Health Service England. NHS strikes major deal to expand hospital capacity to battle coronavirus. 2020 [cited 2021 Feb 10]. Available from: https://www.england.nhs.uk/2020/03/ nhs-strikes-major-deal-to-expand-hospital-capacity-to-battlecoronavirus/

97. Department of Health \& Social Care. UK COVID-19 vaccines delivery plan. 2021 [cited $2021 \mathrm{Feb} 21$ ]. Available from: https:// www.gov.uk/government/publications/uk-covid-19-vaccinesdelivery-plan/uk-covid-19-vaccines-delivery-plan

98. Castro da Rocha FA, Melo LdP, Berenbaum F (2021) Tackling osteoarthritis during COVID-19 pandemic. Ann Rheum Dis 80(2):151-153

99. Arthritis and Musculoskeletal Alliance. Principles for COVID-19 Vaccination in Musculoskeletal and Rheumatology for Clinicians 2021 [cited 2021 Feb 20]. Available from: http://arma.uk.net/ covid-19-vaccination-and-msk/

100. Choi B, Choudhary MC, Regan J et al (2020) Persistence and Evolution of SARS-CoV-2 in an Immunocompromised Host. N Engl J Med 383(23):2291-2293

101. Helleberg M, Niemann CU, Moestrup KS et al (2020) Persistent COVID-19 in an immunocompromised patient temporarily responsive to two courses of remdesivir therapy. J Infect Dis 222(7):1103-1107

102. Chen L, Long X, Xu Q et al (2020) Elevated serum levels of S100A8/A9 and HMGB1 at hospital admission are correlated with inferior clinical outcomes in COVID-19 patients. Cell Mole Immunol 17(9):992-994

103. Zizzo G, Cohen PL (2020) Imperfect storm: is interleukin-33 the Achilles heel of COVID-19? Lancet Rheumatol 2(12):e779-e790

104. Gomez-Cadena A, Spehner L, Kroemer M et al (2021) Severe COVID-19 patients exhibit an ILC2 NKG2D + population in their impaired ILC compartment. Cell Mole Immunol 18(2):484-486

105. Eberl G, Colonna M, Di Santo JP et al (2015) Innate lymphoid cells Innate lymphoid cells a new paradigm in immunology. Science 348(6237):6566

Publisher's Note Springer Nature remains neutral with regard to jurisdictional claims in published maps and institutional affiliations. 